\title{
Article
}

\section{Partial Patellectomy: A Series of 17 Patients}

Balalis, Konstantine, Ziogas, Kleanthis, Tourvas, Evangelos and Topalidou, Anastasia

Available at http://clok.uclan.ac.uk/15577/

Balalis, Konstantine, Ziogas, Kleanthis, Tourvas, Evangelos and Topalidou, Anastasia ORCID: 0000-0003-0280-6801 (2013) Partial Patellectomy: A Series of 17 Patients. Indian Journal of Applied Research, 3 (11). pp. 416-418.

It is advisable to refer to the publisher's version if you intend to cite from the work. $10.15373 / 2249555 X$

For more information about UCLan's research in this area go to

http://www.uclan.ac.uk/researchgroups/ and search for < name of research Group>.

For information about Research generally at UCLan please go to http://www.uclan.ac.uk/research/

All outputs in CLoK are protected by Intellectual Property Rights law, including Copyright law. Copyright, IPR and Moral Rights for the works on this site are retained by the individual authors and/or other copyright owners. Terms and conditions for use of this material are defined in the policies page.

\section{CLoK}

Central Lancashire online Knowledge www.clok.uclan.ac.uk

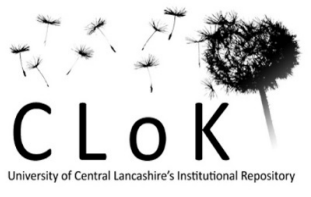




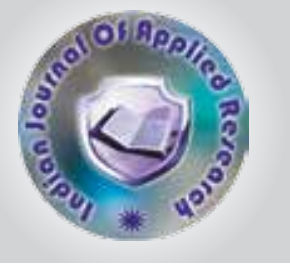

\section{Partial Patellectomy: A Series of 17 Patients}

KEYWORDS

partial patellectomy, comminuted patella fracture, tension band wiring

\section{Konstantine Balalis}

University Hospital of Heraklion, Department of Orthopaedics and Traumatology, Greece

\section{Kleanthis Ziogas}

University Hospital of Heraklion, Department of Orthopaedics and Traumatology, Greece

\section{Evangelos Tourvas}

University Hospital of Heraklion, Department of Orthopaedics and Traumatology, Greece

\section{Anastasia Topalidou}

University Hospital of Heraklion, Department of Orthopaedics and Traumatology, Greece

ABSTRACT The purpose of the study is the evaluation of partial patellectomy in 17 patients with displaced comminuted patella fractures from a total of 146 fractures. Surgical restoration of the knee extension mechanism was supplemented by tension band wired from the patella to the tibial tubercle. The average follow- up period was 26 months. The Cincinatti evaluation form, knee $x$-ray measurements and isokinetic testing was used for the evaluation of all patients. Eleven patients showed very good to excellent results, whereas six patients showed poor to moderate results.

Using magnetic tomography we were able to detect osteoarthritic changes at an early stage. Under given circumstances, partial patellectomy can produce satisfactory outcomes, such as early mobilization and full kinetic recovery within 3 months.

\section{INTRODUCTION}

Even today, the treatment of comminuted patella fractures is a point of much controversy. For a long time, total patellectomy was the chosen therapy for the treatment of such fractures (Brooke, 1937; Duthie \& Hutchinson, 1958; Khong \& Pillay, 1967), but very soon Kaufer (1971) expressed doubts about this method. Today, the preferred therapies are partial patellectomy (Anderson, 1935) and osteosynthesis with tension band (Marya, Bhan \& Dave, 1987). The results of partial patellectomy have since been paralleled with the results obtained using osteosynthesis (Matejcic, Puljiz, Elabjer, Bekavac-Beslin \& Ledinsky, 2008; Dietz, 2009; Della Rocca, 2013) However, non-satisfactory results of partial patellectomy, have also been documented, mainly due to the premature development of patellofemoral arthropathy (Duthie \& Hutchinson, 1958; Hung, Lee, Leung, Chan \& Nicholl, 1993).

The purpose of this study is the evaluation of a series of patients with a variety of clinical and visual representation criteria who had comminuted fractures of the patella, and mostly of the lower patella pole, which couldn't withstand other osteosynthetic method and were treated with the method of partial patellectomy.

\section{MATERIALS AND METHODS}

Between November 2000 and February 2009 we treated 146 skeletally mature patients with patella fractures (Figure 1). Seventeen of those patients were treated with partial patellectomy. These consisted of 11 comminuted fractures and 6 radial fractures, all of which had a large central osseous piece. The classification of the fractures was made using the AO/OTA classification system (Ziegler \& Regazzoni, 1992; Dietz, 2009). The age of the patients was between $28-68$ years old $(47,41 \pm 9,36)$ for the 10 women (W) and 7 men (M) who were included in the study. All patients were Caucasians. We followed clinical progress and re-evaluations for an average of 38 months. The causes of injury were mainly falls $(n=10)$, automobile accidents $(n=4)$, work- related accidents $(n=2)$ and finally sports related accidents $(n=1)$. Fifteen of the patients underwent surgery within the first 24-hour period, and the remaining two within 48 hours.

\subsection{Surgical technique}

The fracture was approached by an anteromedial lengthwise incision of the knee. The lateral and medial retinaculla on both sides were usually ruptured. Following that, a careful removal of all surrounding polar fragments took place, in order to ensure that no bone particles were left behind, checking the condition of the articular cartilage at the same time. With the use of a surgical knife, remaining pieces of bone on the patellar tendon were removed. In two cases of radial fracture, the two main central parts were osteosynthesized using a pair of $\mathrm{K}$ wires. The patellar tendon was then sutured to the patella with an ethibond $\mathbb{1} 1$ suture and pulled out with an entrance point very close to the articular surface, exiting from the front upper surface of the patella on a fully extended knee. A stainless steel wire of $1 \mathrm{~mm}$ diameter was then inserted via a transverse/side tibial tubercle opening, which upon penetrating/crossing the tendon was then tunneled to the upper pole where the two wires end. The stability of this system was checked at $30^{\otimes}, 60^{\otimes}$ and $90^{\circ}$ of flexion. Finally, the lateral and medial retinaculla of the patella and the capsule were sutured and the joint closed (Figure 2). Light immobilization was provided with Robert-Jones bandaging. All patients were encouraged to engage in non-static quadriceps exercises from the first post operative day.

\subsection{Follow- up procedure}

On the third post operative day the passive movement/exercise system (CPM) was applied. Patients were discharged with instructions for a continuation of non-weighted physiotherapy.

Fifteen patients followed the above program, whereas 2 patients with accompanying skin and soft tissue complications had a splint applied. The onset of mobilization for these 2 patients was delayed by a week.

The patients were examined at regular intervals of 1, 3, 6 and 12 months and subsequently once a year.

For the clinical and $x$-ray evaluations, the Cincinnati/Noyes evaluation scales (Crenshaw \& Wilson, 1954) was preferred as the best way of rating patellofemoral findings. The results were therefore characterized as follows: $90-100$ excellent, 80-89 good, 70-79 as moderate and fewer than 70, as poor. 
The $x$-ray examinations included the usual anteroposterior (AP) and lateral (L) radiograph and Merchant's tangent radiograph. Insall-Salvati ratio was used for the assessment of patellar height. Muscle atrophy was observed with measurement of the perimeter of the quadriceps muscles using the healthy muscle as a comparison. Six patients also underwent an MRI knee tomography.

\subsection{Statistical analysis}

Because of the small amount of patients, we used the method of descriptional statistics.

\section{RESULTS}

\subsection{Subjective evaluation:}

Using the Cincinnati evaluation scale in the 3 months re-evaluation, 7 patients showed excellent results (41\%), 4 showed good results $(23,5 \%), 4$ showed moderate results $(23,5 \%)$, and 2 showed poor results (12\%). These results showed a significant improvement at 6 months.

At 6 months reevaluation ten patients (59\%) reported knee discomfort, 8 of which after medium exertion, 2 with continuous pain and reduction of activity. Three patients (18\%) complained of knee swelling and a certain amount of movement restriction while working, taking part in sports, or during everyday activities. At the last follow-up examination, 3 patients (18\%) complained of a "giving way" sensation.

\subsection{Objective evaluation}

Range Of Motion (ROM): For 14 patients ROM was above $90 \%$ of the norm at a very early stage ( 3 months examination). This resulted till the end of the evaluation period. For 2 patients, the final ROM was $80-90 \%$ and for 1 patient less than $70 \%$, who also had a $10 \%$ loss of extension and an obvious limp.

Muscle atrophy: Three patients had quadriceps atrophy at 1 and 3 months re-evaluation.

Grind Test: From the total of 17 patients that were examined, 8 patients (47\%) showed a positive patella pressure point.

\subsection{X-Ray Evaluation}

X-rays showed that in 10 patients (59\%) patellofemoral arthropathy was present at an early stage or already established ( 3 and 6 months re- evaluation). In 6 of these patients medial compartment arthropathy (35\%) was also observed, and in 2 in both compartments (12\%).

In the Merchant position, 4 knees showed a post operative change of the patellar pulley relationship (3 months). Of those, during re-evaluations, 1 showed improvement, while the other 3 developed osteoarthritic changes $(17,5 \%)$.

Insall-Salvati ratio: Patella tendon's length/ patella's length $(A B)=0.9$ (mean value)

Six patients with moderate and poor results after 6 months underwent an MRI examination, which showed development or established osteoarthritic changes. Moreover, slight ossification of the quadriceps tendon presented in 2 patients.

\subsection{Complications}

In the immediate complications were included 2 superficial infections, which were treated successfully with antibiotics. No wire breakage was observed within the immediate post operative period. Fourteen "silent" breaks resulted after the first 3 months, although exact breakage time could not be ascertained (Figure 3).

\section{DISCUSSION}

Despite the results of a previous study that suggested that $20-25 \%$ of patella fractures undergo partial patellectomy (Hung et al., 1993), in our series, only $11,6 \%$ of patella frac- tures underwent this procedure.

The use of tension band wiring as a safety measure for early mobilization has not made the method more popular and widely used. Although many authors (Nummi, 1971; Akeson, Amiel, Abel, Garfin \& Woo, 1987) had some good results in the past, then came the research of Marder, Swanson, Sharkey and Duwelius (1993) creating an atmosphere of discouragement for the method. Our findings corroborate the above controversies with $64 \%$ of good or excellent results.

In a further analysis of the results, we noticed that in knees undergoing only partial patellectomy using the method of reinforcement with tension band wiring, mobility was quickly achieved, as was the recovery of muscle strength which was both quick and complete. According to Akeson et al. (1987), immobilization is a negative effect for all joints and especially for the cartilage. Therefore, the beneficial influence of early mobilization on the cartilage was also added to the advantages in our research findings.

The reduction of muscle strength in total patellectomy reaches 50\% (Watkins, Harris, Wender, Zarins \& Rowe, 1983), whereas only $15 \%$ in partial patellectomy (Saltzman, Goulet, McClellan, Schneider \& Matthews, 1990).

For the development of osteoarthritic changes, the results of our series are comparable to those of Saltzman et al. (1990) in which, nevertheless, the early mobilization was not introduced. It would seem that early mobilization does not play a role in producing osteoarthritic changes. Probably, the nature and position of cartilage is a significant factor in the development of secondary changes (Hung et al., 1993).

While investigating other factors which predispose towards osteoarthritic changes, we have not take into account the size and placing of the patella abutment. Attempts have already been made by many authors (Goodfellow, Hungerford \& Zindel, 1976; Aglietti, Insall \& Cerulli, 1983; Huberti \& Hayes, 1984) to map patella pressure points on a pulley at varying flexion degrees. Undoubtedly, the low positioning of the patella and the partial resection contribute to the increase in patellar pressure and quite possibly these changes account for the development of secondary osteoarthritic changes similarly indicated by another study (Hampson \& Hill, 1975). This phenomenon was also observed in our series and is in accordance with the Marder et al (1993) study which also adds that patellofemoral contact can be relieved by a frontal abutment positioning despite the prevailing current options (Duthie \& Hutchinson, 1958). In our study, posterior positioning was used with only $64 \%$ satisfactory results.

\section{CONCLUSION}

In conclusion, if the above conditions, in relation to the large abutment size, placement and anterior tendon placement on the abutment are met, then partial patellectomy gives satisfactory results. Otherwise an attempt of osteosynthesis should be made, maintaining the patella. In any case, disorders of the articular surface $<2 \mathrm{~mm}$ are acceptable with satisfactory results. 


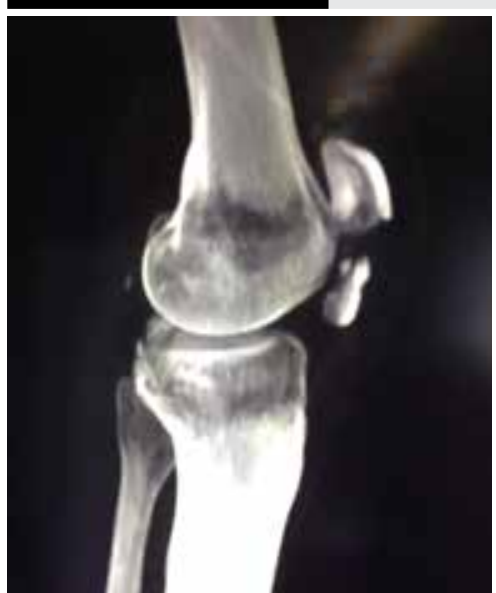

Figure 1. Preoperative CT scan which demonstrates the patellar fracture with an interior bone fragment which attaches to the quadriceps tendon.

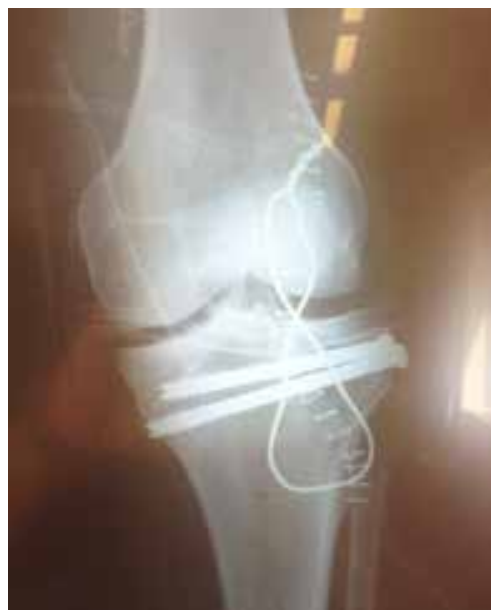

Figure 2. Surgical treatment with the use of tension band wiring for the remaining piece of the patella.

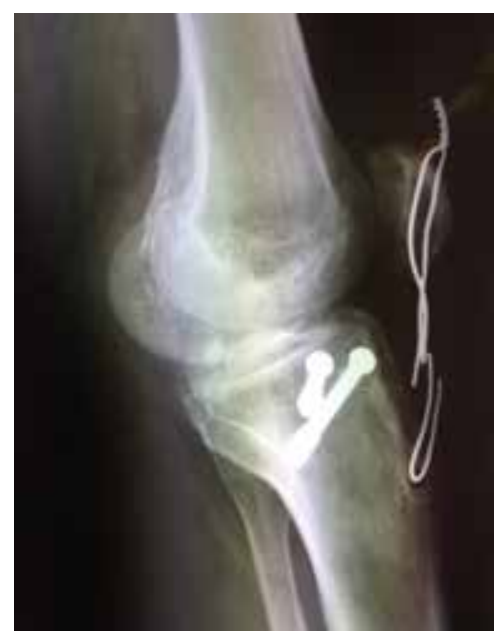

Figure 3. Breakage of the wire

REFERENCE 1. Aglietti, P., Insall, J.N., \& Cerulli, G. (1983). Patellar pain and incongruence I: Measurements of incongruence. Clin Orthop Relat Res, 176 , 217-224. |2. Akeson, W.H. Amiel, D. Abel, M.F., Garfin, S.R., \& Woo, S.L. (1987). Effects of immobilization on joints. Clin Orthop Relat Res, 219 28-37. 3. Anderson, R (1935). An ambulatory method of treating fractures of the patella. Ann Surg, 101(4), 1082-1090.|4. Brooke R. (1937). The Effect of Removal of the Patella for Simple Transverse Fractures on the Function of the Knee-joint (Section of Orthopædics). Proc R Soc Med, 30(3), 203-207. | 5. Crenshaw, A.H., \& Wilson, F.D. (1954). The surgical treatment of fractures of the patella. South Med J, 47 (8), 716-720. | 6. Della Rocca, G.J. (2013). Displaced patella fractures. J Knee Surg, 26(5), 293-300. | 7. Dietz, S.O., Hessmann, M.H., Gercek, E., \& Rommens, P.M. (2009). Patella fracture. Oper Orthop Traumatol, 21(2), 206-220. | 8. Duthie, H.L., \& Hutchinson, J.R. (1958). The results of partial and total excision of the patella. J Bone Joint Surg Br, 40-B(1), 75-81. | 9. Goodfellow, J., Hungerford, D.S., Zindel, M. (1976). Patello-femoral joint mechanics and pathology 1: Functional anatomy of the patello-femoral joint. J Bone Joint Surg Br, 58(3), 287-90. | 10. Hampson, W.G., \& Hill, P. (1975). Late results of transfer of the tibial tubercle for recurrent dislocation of the patella. J Bone Joint Surg Br, 57(2), 209-213. | 11. Huberti, H.H., \& Hayes, W.C. (1984). Patellofemoral contact pressures. The influence of $q$-angle and tendofemoral contact. J Bone Joint Surg Am, 66(5), 715-724 | 12. Hung, L.K., Lee, S.Y., Leung, K.S., Chanm, K.M., \& Nicholl, L.A. (1993). Partial patellectomy for patellar fracture: tension band wiring and early mobilization. J Orthop Trauma, 7(3), 252-260. | 13. Kaufer, H. (1971). Mechanical Function of the Patella. J Bone Joint Surg Am, 53(8), 1551-1560. | 14. Khong, B, T., \& Pillay, V.K. (1967). Patellectomy for fracture. A study of the results of 40 cases. Singapore Med J, 8(3), 230-234. | 15. Marder, R.A., Swanson, T.V., Sharkey, N.A., \& Duwelius, P.J. (1993). Effects of partial patellectomy and reattachment of the patellar tendon on patellofemoral contact areas and pressures. J Bone Joint Surg Am, 75(1), 35-45. | 16. Marya, S.K., Bhan, S., \& Dave, P.K. (1987). Comperative study of knee function after patellectomy and osteosynthesis with a tension band wire following patellar fractures. Int Surg, $72(4), 211-213$. | 17. Matejcic, A., Puljiz, Z., Elabjer, E., Bekavac-Beslin, M., \& Ledinsky, M. (2008) Multifragment fracture of the patellar apex: basket plate osteosynthesis compared with partial patellectomy. Arch Orthop Trauma Surg, 128(4), 403-408. | 18. Nummi, J. (1971). Operative treatment of patellar fractures. Acta Orthop Scand, 42(5), 437-438. 19. Saltzman, C.L., Goulet, J.A., McClellan, R.T., Schneider, L.A., \& Matthews, L.S. (1990). Results of treatment of displaced patellar fractures by partial patellectomy. J Bone Joint Surg Am, 72(9), 1279-1285. | 20. Watkins, M.P., Harris, B.A., Wender, S., Zarins, B., \& Rowe, C.R. (1983). Effect of patellectomy on the function of the quadriceps and hamstrings. J Bone Joint Surg Am, 65(3), 390-395. |21. Ziegler, J.P., \& Regazzoni, P. (1992). Results of treatment of patellar fractures. Helv Chir Acta, 58(6), 949-952. | 